\title{
Thaine's method for circular units and a conjecture of Gross
}

\author{
Henri Darmon
}

September 9, 2007

\section{Introduction}

This paper formulates a refined analogue of the usual class number formula for a real quadratic extension of $\mathbf{Q}$, using circular units. The statement of this conjecture is inspired by an analogous conjecture of Gross [Gr]. Strong evidence for this conjecture can be given thanks to F. Thaine's powerful method $[\mathrm{Th}]$ for generating relations in ideal class groups using circular units.

The first two sections briefly recall Dirichlet's analytic class number formula and Gross's refinement of it; they are there mainly to fix notations and provide motivation. Section 4 states the new conjecture. The remaining sections are devoted to proving various results that support it.

Acknowledgements: I wish to thank Massimo Bertolini and Benedict Gross for many stimulating conversations on the topics of this paper. This work was supported at different stages by a Sloan Doctoral Dissertation fellowship, an NSERC postdoctoral felloswhip, and by NSF grant \# DMS-8703372.A04.

Notations: If $K$ is a number field and $w$ is a place of $K$ lying above a prime $v$ of $\mathbf{Q}$, we denote by $K_{w}$ the localization of $K$ at $w$, and let $\mathbf{N} w$ be the order of its residue field. The $w$-adic norm \|\|$_{w}$ is normalized so that it is equal to $\mathbf{N} w^{-1}$ on uniformizing elements.

Given a finite abelian extension $M / K$, we let

$$
\operatorname{rec}_{w}: K_{w}^{*} \longrightarrow \operatorname{Gal}(M / K)
$$


denote the reciprocity map of local class field theory. When $w$ is unramified in $M / K$, it factors through the valuation map $K_{w}^{*} \longrightarrow \mathbf{Z}$ and maps uniformizing elements to $\operatorname{Frob}_{w}$, the Frobenius element in $\operatorname{Gal}(M / K)$ characterized by

$$
\operatorname{Frob}_{w}(x)=x^{\mathbf{N} w} \quad(\bmod \tilde{w}),
$$

where $\tilde{w}$ is any place of $M$ above $w$.

We write $\operatorname{Div}(K)$ for the free $\mathbf{Z}$-module generated by the finite places of $K$, and $P(K)$ for the submodule generated by the principal divisors. The class group $C(K)$ is the quotient $\operatorname{Div}(K) / P(K)$. Given a set $S$ of places of $K$, let $\langle S\rangle$ be the $\mathbf{Z}$-span of the elements of $S$ in $\operatorname{Div}(K)$, and let

$$
C_{S}(K)=\langle S\rangle \backslash \operatorname{Div}(K) / P(K) .
$$

\section{Dirichlet's analytic class number formula}

We recall briefly the analytic class number formula of Dirichlet relating the behavior of the $L$-series of a number field at $s=0$ to the arithmetic properties of that number field. The exposition follows closely the one in $[\mathrm{Gr}]$.

Let $K$ be a number field, and choose a finite set $S$ of places of $K$ containing all of the archimedean places. Let $T$ be a finite set of places of $K$ disjoint from $S$.

There is associated to this situation the local data which describes the splitting of the primes in $K$. This data is conveniently encoded in the Euler product

$$
L_{S, T}(K, s)=\prod_{v \notin S}\left(1-\mathbf{N} v^{-s}\right)^{-1} \prod_{v \in T}\left(1-\mathbf{N} v^{1-s}\right) .
$$

Here the products are taken over the non-archimedean places of $K$. The Euler product defines the $L$-function $L_{S, T}(K, s)$ in some right half plane of convergence, and it is known that $L_{S, T}(K, s)$ has a meromorphic continuation to the entire complex plane.

The number field $K$ together with the sets $S$ and $T$ gives rise to more subtle global invariants.

1. The group $\left(\mathcal{O}_{S}^{*}\right)_{T}$ of $S$-units which are congruent to 1 modulo the places of $T$. This is a finitely generated abelian group which is free when $T$ is large enough. Let $r$ denote the rank of this group. By Dirichlet's unit theorem, one has $r=\#(S)-1$. 
2. The torsion subgroup $\left[\left(\mathcal{O}_{S}^{*}\right)_{T}\right]_{\text {torsion }}$ which is cyclic of order $w_{S, T}$. (Typically we will choose $T$ so that $w_{S, T}=1$.)

3. The Picard group $\operatorname{Pic}\left(\mathcal{O}_{S}\right)_{T}$ of invertible $\mathcal{O}_{S}$-modules together with a trivialization at $T$. It is a finite extension of $C_{S}(K)$. Let $h_{S, T}$ denote its order.

4. The $S$-unit regulator $R_{S, T}$, defined as follows. Let $X=\operatorname{Div}^{0}(S)$ be the free abelian group generated by the formal linear combinations of places of $S$ of degree 0 ,

$$
X=\left\{\sum_{v \in S} n_{v} v, \quad \sum n_{v}=0\right\} .
$$

The logarithmic embedding $\log _{S}: \mathcal{O}_{S}^{*} \longrightarrow \mathbf{R} \otimes X$ of the $S$-units is defined by

$$
\log _{S}(u)=\sum_{v \in S} \log \|u\|_{v} \otimes v .
$$

Both $\left(\mathcal{O}_{S}^{*}\right)_{T}$ and $X$ are of rank $r$. Let

$$
\Lambda^{r} \log _{S}: \Lambda^{r} \mathcal{O}_{S}^{*} \longrightarrow \Lambda^{r}(\mathbf{R} \otimes X)
$$

denote the map induced by $\log _{S}$ on the top exterior powers, and define the regulator $R_{S, T}$ by

$$
\Lambda^{r} \log _{S}\left(\gamma_{1} \wedge \cdots \wedge \gamma_{r}\right)=R_{S, T} \otimes\left(v_{1} \wedge \cdots \wedge v_{r}\right)
$$

where $\gamma_{1}, \ldots, \gamma_{r}$ (resp. $\left.v_{1}, \ldots, v_{r}\right)$ are integral bases for $\left(O_{S}^{*}\right)_{T}$ modulo torsion (resp. $X$ ), normalized so that $R_{S, T}$ is positive.

The theorem of Dirichlet asserts that the above global invariants appear in the Taylor expansion of the $L$-function $L_{S, T}(K, s)$ which was constructed using purely local data. It is one of the simplest manifestations of a local global principle which is pervasive in number theory.

\section{Theorem 2.1 (Dirichlet)}

1. The L-series $L_{S, T}(K, s)$ vanishes to order $r$ at $s=0$.

2. The Taylor expansion of $L_{S, T}(K, s)$ at $s=0$ is given by:

$$
L_{S, T}(K, s)=-\frac{h_{S, T} R_{S, T}}{w_{S, T}} s^{r}+O\left(s^{r+1}\right) .
$$




\section{Gross's refined class number formula}

We now turn to the refined class number formula of Gross, following closely the account given in $[\mathrm{Gr}]$.

Let $L$ be a finite abelian extension of $K$ which is unramified outside the places of $S$, and let $G=\operatorname{Gal}(L / K)$. Define a complex-valued function $\hat{\theta}_{G}$ on the dual group $\hat{G}=\operatorname{hom}\left(G, \mathbf{C}^{*}\right)$ by

$$
\hat{\theta}_{G}(\chi)=L_{S, T}(K, \chi, 0),
$$

where, for a complex character $\chi: G \longrightarrow \mathbf{C}^{*}$ and a complex number $s$ with $\Re s>1$, the complex function $L_{S, T}(K, \chi, s)$ is defined by the convergent Euler product

$$
L_{S, T}(K, \chi, s)=\prod_{v \notin S}\left(1-\chi\left(\operatorname{Frob}_{v}\right) \mathbf{N} v^{-s}\right)^{-1} \prod_{v \in T}\left(1-\chi\left(\operatorname{Frob}_{v}\right) \mathbf{N} v^{1-s}\right) .
$$

This function has a meromorphic continuation to the entire complex plane and is regular at $s=0$. Let $\theta_{G} \in \mathbf{C}[G]$ be the Fourier transform of $\hat{\theta}_{G}$,

$$
\theta_{G}=\sum_{\chi \in \hat{G}} \hat{\theta}_{G}(\chi) e_{\chi}, \quad e_{\chi}=1 /|G| \sum_{g \in G} \chi(g) g^{-1} .
$$

Thus, $\theta_{G}=\sum_{g \in G} a(g) g$ interpolates values of $L_{S, T}(K, \chi, 0)$,

$$
\sum_{g \in G} a(g) \chi(g)=L_{S, T}(K, \chi, 0) .
$$

For the rest of this section, we make the following assumption on $T$, which forces $w_{S, T}=1$ so that the leading term in the class number formula is integral.

Hypothesis 3.1 Suppose that $T$ contains two primes of unequal residue characteristic, or that $T$ contains a prime whose absolute ramification index in $K$ is strictly less that the residue field characteristic minus 1.

Under this condition, Gross $[\mathrm{Gr}]$ shows that the element $\theta_{G}$ belongs to the integral group ring $\mathbf{Z}[G]$.

Fact 3.2 (Gross) $\theta_{G}$ belongs to $\mathbf{Z}[G]$. 
The order of vanishing of $\theta_{G}$ : Let $I$ denote the augmentation ideal in the group ring $\mathbf{Z}[G]$. It is the kernel of the augmentation homomorphism $\epsilon$ : $\mathbf{Z}[G] \longrightarrow \mathbf{Z}$ which sends $\sigma \in G$ to 1 . The powers $I \supset I^{2} \supset \cdots$ define a decreasing filtration on $\mathbf{Z}[G]$. Because of the exact sequence

$$
0 \longrightarrow I \longrightarrow \mathbf{Z}[G] \stackrel{\epsilon}{\longrightarrow} \mathbf{Z} \longrightarrow 0,
$$

one has $\mathbf{Z}[G] / I=\mathbf{Z}$. The higher quotients in the filtration are torsion. For instance, there is a natural homomorphism $G \longrightarrow I / I^{2}$ which sends $\sigma \in G$ to $\sigma-1\left(\bmod I^{2}\right)$. In fact, this is an isomorphism. More generally, there is a natural surjective map

$$
\operatorname{Sym}^{r}(G) \longrightarrow I^{r} / I^{r+1}
$$

which sends $\sigma_{1} \otimes \cdots \otimes \sigma_{r}$ to $\left(\sigma_{1}-1\right) \cdots\left(\sigma_{r}-1\right) \quad\left(\bmod I^{r+1}\right)$. (This map is not necessarily an isomorphism; for a detailed study of the map $\operatorname{Sym}(G) \longrightarrow$ $\oplus_{r} I^{r} / I^{r+1}$, the reader may consult [Pa], [H1], [H2].)

The element $\theta_{G}$ which interpolates special values at $s=0$ of the twisted $L$-function $L_{S, T}(K, \chi, s)$ is what plays the role of the $L$-function in Gross's refined class number formula. To say that this element vanishes to order $r$ is to say that it belongs to the $r$-th power of the augmentation ideal.

Conjecture 3.3 (Gross) The element $\theta_{G}$ belongs to $I^{r}$.

The leading coefficient $\tilde{\theta}_{G}$ in the refined class number formula is defined to be the projection of $\theta_{G}$ to $I^{r} / I^{r+1}$. It is natural to search for an interpretation of $\tilde{\theta}_{G}$ which is analogous to the analytic result of Dirichlet.

To do this, it suffices to change the definition of the regulator term $R_{S, T}$ defined in the previous section. Consider the homomorphism

$$
\operatorname{rec}_{S}: \mathcal{O}_{S}^{*} \longrightarrow\left(I / I^{2}\right) \otimes_{\mathbf{z}} X
$$

defined by

$$
\operatorname{rec}_{S}(u)=\sum_{v \in S}\left(\operatorname{rec}_{v}\left(u_{v}\right)-1\right) \otimes v,
$$

where $u_{v} \in K_{v}^{*}$ is the natural image of $u$. Let $\Lambda^{r} \operatorname{rec}_{S}$ denote the induced map on the top exterior powers:

$$
\Lambda^{r} \operatorname{rec}_{S}: \Lambda^{r} \mathcal{O}_{S}^{*} \longrightarrow \Lambda^{r}\left(I / I^{2} \otimes X\right) \longrightarrow\left(I^{r} / I^{r+1}\right) \otimes \Lambda^{r} X,
$$


and define the regulator $R_{S, T}$ in $I^{r} / I^{r+1}$ by

$$
\Lambda^{r} \operatorname{rec}_{S}\left(\gamma_{1} \wedge \cdots \wedge \gamma_{r}\right)=R_{S, T} \otimes\left(v_{1} \wedge \cdots \wedge v_{r}\right),
$$

where $\gamma_{1}, \ldots, \gamma_{r}$ and $v_{1}, \ldots, v_{r}$ are the integral bases chosen in section 2 .

\section{Conjecture 3.4 (Gross)}

$$
\tilde{\theta}_{G}=-h_{S, T} R_{S, T}
$$

\section{Remarks:}

1 . If $K$ has a complex place $v$, then the $\Gamma$-factors in the functional equation force a zero at $s=0$ in the twisted $L$-function $L_{S, T}(K, \chi, s)$ for all $\chi$. Hence $\theta_{G}=0$. But rec $v$ is trivial, so that $R_{S, T}=0$ as well. Therefore the conjecture is trivially verified. It is only interesting when $K$ is a totally real field.

2. Because of the presence of the archimedean places, one has $2 R_{S, T}=0$ in $I^{r} / I^{r+1}$. (Also one can show that $2 \tilde{\theta}_{G}=0$.) Thus Gross's conjecture for number fields is really a parity statement - it was proved by Gross when $S$ contains only the archimedean places by using the 2-adic congruences of Deligne-Ribet for totally real fields [DR].

\section{A refined conjecture for circular units}

Let $\omega$ be an even primitive Dirichlet character of conductor $N$. In order to simplify the exposition, we assume that $\omega$ is quadratic, and let $K$ denote the corresponding real quadratic field. Choose an auxiliary real abelian extension $M$ of $\mathbf{Q}$ with conductor prime to $N$, and let $G$ denote its Galois group. For all $\chi$ in $\hat{G}$, the Dirichlet $L$-series

$$
L_{S}(s, \omega \chi)=\sum_{(n, S)=1}^{\infty} \omega \chi(n) n^{-s}=\prod_{p \mid S}\left(1-\omega \chi(p) p^{-s}\right)^{-1}
$$

vanishes at $s=0$, because of the pole in the factor $\Gamma\left(\frac{1}{2} s\right)$ in the functional equation. One might be tempted to define a function $\hat{\theta}_{G}^{\prime}$ on $\hat{G}$ by $\hat{\theta}_{G}^{\prime}(\chi)=$ $L_{S}^{\prime}(0, \omega \chi)$, and letting $\theta_{G}^{\prime} \in \mathbf{C}[G]$ be its Fourier transform as in section 3 . However, the coefficients of $\theta_{G}^{\prime}$ are not integral, or even algebraic. This leads to the problem of finding an appropriate substitute for $\theta_{G}^{\prime}$, and formulating a conjecture analagous to conjectures 3.3 and 3.4 for it. 
Fix a choice of primitive $n$th roots of unity $\zeta_{n} \in \overline{\mathbf{Q}}$ for each $n$, satisfying the compatibilities

$$
\zeta_{n m}^{m}=\zeta_{n}
$$

This choice determines a complex embedding $\Psi$ of $\mathbf{Q}^{a b}$, sending $\zeta_{n}$ to $e^{2 \pi i / n}$.

Let $S$ be a square-free integer which is relatively prime to the conductor of $\omega$. Let $K_{S}=K\left(\mu_{S}\right)$. The circular unit $\alpha_{S}$ in $K_{S}$ is defined by

$$
\alpha_{S}=\prod_{\sigma \in \operatorname{Gal}\left(\mathbf{Q}\left(\mu_{S N}\right) / \mathbf{Q}\left(\mu_{S}\right)\right)} \sigma\left(\zeta_{N S}-1\right)^{\omega(\sigma)}
$$

Let $\Gamma_{S}=\operatorname{Gal}\left(K_{S} / K\right)$, and let $I$ denote the augmentation ideal in the group ring $\mathbf{Z}\left[\Gamma_{S}\right]$. The theta-element $\theta^{\prime}(\omega, S)$ is given by the formula

$$
\theta^{\prime}(\omega, S)=\sum_{\sigma \in \Gamma_{S}} \sigma \alpha_{S} \otimes \sigma \quad \in K_{S}^{*} \otimes \mathbf{Z}\left[\Gamma_{S}\right]
$$

Relation between $\theta^{\prime}(\omega, S)$ and $L_{S}^{\prime}(0, \omega \chi)$ : Let $\log : K_{S}^{*} \longrightarrow \mathbf{C}$ be a principal branch of the logarithm map induced by the complex embedding $\Psi$ of $K_{S}$. Extending a character $\chi \in \hat{\Gamma}_{S}$ by linearity to the group ring $\mathbf{Z}\left[\Gamma_{S}\right]$, one combines the maps $\log$ and $\chi$ to give a linear map

$$
\log \otimes \chi: K_{S}^{*} \otimes \mathbf{Z}\left[\Gamma_{S}\right] \longrightarrow \mathbf{C} .
$$

We call a character $\chi$ of $\Gamma_{S}$ primitive if it does not factor through the natural homomorphism $\Gamma_{S} \longrightarrow \Gamma_{T}$ for any proper divisor $T$ of $S$. The following theorem which describes the interpolation property of the circular units is due to Kummer.

Theorem 4.1 Assume that $\chi$ is primitive. Then

$$
\log \otimes \chi\left(\theta^{\prime}(\omega, S)\right)=\sum_{\sigma \in \Gamma_{S}} \chi(\sigma) \log \left|\sigma \alpha_{S}\right|=-2 L_{S}^{\prime}(0, \omega \chi) .
$$

Thus $\theta^{\prime}(\omega, S)$ can be viewed as an analogue of $L_{S}^{\prime}(s, \omega)$.

Let

$$
\begin{aligned}
S_{\text {split }} & =\{l \mid S, \quad \omega(l)=1\} \\
S_{\text {inert }} & =\{l \mid S, \quad \omega(l)=-1\} .
\end{aligned}
$$


Let $X^{-}$be the group of divisors of $K$ of degree 0 lying above $S$ or $\infty$ on which the generator of $\operatorname{Gal}(K / \mathbf{Q})$ acts by -1 . It is a free $\mathbf{Z}$-module of rank $r$, where

$$
r=\#\left(S_{\text {split }}\right)+1 \text {. }
$$

Let $v_{\infty}=\lambda_{\infty}-\bar{\lambda}_{\infty}$ be the difference of the two conjugate real places of $K$, and let $v_{i}=\lambda_{i}-\bar{\lambda}_{i}$, where $\lambda_{i}, \bar{\lambda}_{i}$ denote conjugate primes of $K$ lying above $l_{i} \in S_{\text {split }}$. Then $\left\{v_{\infty}, v_{1}, \ldots, v_{r-1}\right\}$ forms a basis for $X^{-}$.

Let $\left(\mathcal{O}_{S}^{*}\right)^{-}$be the group of $S$-units of $K$ on which the generator of $\operatorname{Gal}(K / \mathbf{Q})$ acts by -1 . This is also a free $\mathbf{Z}$-module of rank $r$. Choose a basis $\omega_{1}, \ldots, \omega_{r}$ for $\left(\mathcal{O}_{S}^{*}\right)^{-}$in such a way that the regulator $R_{S}$ for the logarithmic embedding

$$
\left(\mathcal{O}_{S}^{*}\right)^{-} \longrightarrow X^{-} \otimes \mathbf{R}
$$

relative to the bases $\left\{\omega_{1}, \ldots, \omega_{r}\right\}$ and $\left\{v_{\infty}, v_{1}, \ldots, v_{r-1}\right\}$ is positive.

From the non-vanishing of the classical Dirichlet $L$-series at $s=1 \mathrm{com}$ bined with the functional equation for these $L$-series, one knows that

$$
\operatorname{ord}_{s=0} L_{S}^{\prime}(s, \omega)=r-1,
$$

and that

$$
\lim _{s \rightarrow 0} L_{S}^{\prime}(s, \omega) /\left(s^{r-1}\right)=-2^{\# S_{\text {inert }}+1} r h_{S} R_{S} .
$$

In the next section, we will show that a similar statement is true for the element $\theta^{\prime}(\omega, S)$ :

Theorem 4.2 (Order of vanishing) The element $\theta^{\prime}(\omega, S)$ belongs to the group $K_{S}^{*} \otimes I^{r-1}$.

The leading coefficient $\tilde{\theta}^{\prime}(\omega, S)$ is defined to be the natural projection of $\theta^{\prime}(\omega, S)$ to the group $K_{S}^{*} \otimes\left(I^{r-1} / I^{r}\right)$. One can interpret $\theta^{\prime}(\omega, S)$ by means of a kind of $S$-unit regulator belonging to $\mathcal{O}_{S}^{*} \otimes\left(I^{r-1} / I^{r}\right)$.

The regulator: Let $Y^{-}$denote the group of divisors of $K$ of degree 0 lying above $S$ on which $\operatorname{Gal}(\overline{\mathbf{Q}} / \mathbf{Q})$ acts via the character $\omega$. This is a free module of rank $r-1$ with basis $\left\{v_{1}, \ldots, v_{r-1}\right\}$. One defines the map

$$
\operatorname{rec}_{S}:\left(\mathcal{O}_{S}^{*}\right)^{-} \longrightarrow I_{S} \otimes Y^{-}
$$

using the reciprocity law of local class field theory as in section 3 . Define the partial regulators $R_{i} \in I_{S}^{r-1} / I_{S}^{r}$ by the formula

$$
\operatorname{rec}_{S}\left(\gamma_{1} \wedge \cdots \wedge \gamma_{i-1} \wedge \gamma_{i+1} \wedge \cdots \wedge \gamma_{r}\right)=R_{i} \otimes\left(v_{1} \wedge \cdots \wedge v_{r-1}\right) .
$$


The regulator $R_{S} \in \mathcal{O}_{S}^{*} \otimes\left(I^{r-1} / I^{r}\right)$ is given by

$$
R_{S}=\sum_{i=1}^{r}(-1)^{i+1} \gamma_{i} \otimes R_{i}
$$

\section{Conjecture 4.3}

$$
\tilde{\theta}^{\prime}(\omega, S)=-2^{\#\left(S_{\text {inert }}\right)+1} h_{S} R_{S}
$$

We now give some evidence for conjecture 4.3. Let $\tilde{\theta}^{\prime}(\omega, S)_{2}$ denote the projection of $\tilde{\theta}^{\prime}(\omega, S)$ in the group $K_{S}^{*} \otimes\left(I_{2}^{r-1} / I_{2}^{r}\right)$, where $I_{2}$ denotes the augmentation ideal in the group ring $\mathbf{Z}\left[\frac{1}{2}\right]\left[\Gamma_{S}\right]$. The tensoring with the ring $\mathbf{Z}\left[\frac{1}{2}\right]$ has been made to avoid some technical complications associated with the prime 2: observe that $\left(I_{2}^{r-1} / I_{2}^{r}\right)=\left(I^{r-1} / I^{r}\right) \otimes \mathbf{Z}\left[\frac{1}{2}\right]$ is a finite abelian group of odd order, when $r>1$.

Fact 4.4 The natural map $K^{*} \otimes\left(I_{2}^{r-1} / I_{2}^{r}\right) \longrightarrow K_{S}^{*} \otimes\left(I_{2}^{r-1} / I_{2}^{r}\right)$ is an injection.

The proof for this standard fact will be given in section 9 .

Let $n(S)$ be the greatest odd divisor of $\operatorname{gcd}_{l \mid S}(l-1)$. The following theorem gives some evidence for conjecture 4.3:

\section{Theorem 4.5 .}

1. Conjecture 4.3 is true when $r=1$.

2. $\tilde{\theta}^{\prime}(\omega, S)_{2}$ belongs to $K^{*} \otimes I_{2}^{r-1} / I_{2}^{r}$.

3. If $\operatorname{gcd}\left(h_{S}(K), n(T)\right)=1$ for all $T \mid S$, then $\tilde{\theta}^{\prime}(\omega, S)_{2}$ belongs to $\mathcal{O}_{s}^{*} \otimes$ $\left(I_{2}^{r-1} / I_{2}^{r}\right)$.

4. $h_{S}(K)$ divides $\tilde{\theta}^{\prime}(\omega, S)_{2}$.

5. Suppose that $\Gamma_{S}=\Gamma_{l}$ is cyclic, and that $l$ is split in $K / \mathbf{Q}$ so that $r=2$. Let $\lambda$ be a prime of $K$ above $l$, and let $k_{\lambda} \simeq \mathbf{F}_{l}$ denote the residue field at $\lambda$. If the fundamental unit of $K / \mathbf{Q}$ is a generator for $k_{\lambda}^{*}$, and $\operatorname{gcd}(h(K), n(l))=1$, then

$$
\tilde{\theta}^{\prime}(\omega, l)_{2}= \pm 2 h_{l} R_{l} \quad\left(\bmod I_{2}^{2}\right) .
$$

The proof of this theorem, which uses the methods of Thaine [Th] in an essential way, will be given in section 9 


\section{The Euler system of circular units}

Let $\mathcal{S}$ be the set of square-free integers prime to the conductor of $K$. For all $S \in \mathcal{S}$ we are given the following data:

1. An abelian extension $K_{S}=K\left(\mu_{S}\right)$ of $K$ with Galois group $\Gamma_{S}=$ $(\mathbf{Z} / S \mathbf{Z})^{*}$.

2. The circular unit $\alpha(S)$ in $K_{S}$, given by the formula

$$
\alpha(S)=\prod_{\sigma \in \operatorname{Gal}\left(\mathbf{Q}\left(\zeta_{S N}\right) / \mathbf{Q}\left(\zeta_{S}\right)\right)} \sigma\left(\zeta_{S N}-1\right)^{\omega(\sigma)} .
$$

Writing $S=l_{1} \cdots l_{s}$, the extension $K_{S}$ is a compositum of the fields $K_{l_{i}}$ which are linearly disjoint over $K$. Hence there is a canonical direct product decomposition

$$
\Gamma_{S}=\Gamma_{l_{1}} \times \cdots \times \Gamma_{l_{s}}
$$

which gives inclusions $\Gamma_{T} \subset \Gamma_{S}$ for all divisors $T$ of $S$. We will implicitly identify elements of $\Gamma_{T}$ with their images in $\Gamma_{S}$. For any $T$ dividing $S$, the partial norm operator $\mathbf{N}_{T}$ in the group ring $\mathbf{Z}\left[\Gamma_{S}\right]$ is defined by

$$
\mathbf{N}_{T}=\sum_{\sigma \in \Gamma_{T}} \sigma
$$

These operators act on the field $K_{S}$ in the natural way. Given $T \in \mathcal{S}$ and $l$ a prime in $\mathcal{S}$ which is prime to $T$, let $\sigma_{l, T} \in \operatorname{Gal}\left(K_{T} / \mathrm{Q}\right)$ be the automorphism sending the roots of unity to their $l$ th powers.

\section{Proposition 5.1}

$$
N_{l}(\alpha(T l))=\left(1-\sigma_{l, T}^{-1}\right) \alpha(T) .
$$

Proof: We can write

$$
\zeta_{T l}=\zeta_{T}^{a} \zeta_{l}^{b}
$$

where $a l+b T=1$. Hence

$$
N_{l}\left(1-\zeta_{T l}\right)=\left(1-\zeta_{T}^{a l}\right) /\left(1-\zeta_{T}^{a}\right)=\left(1-\sigma_{l, T}^{-1}\right)\left(1-\zeta_{T}\right),
$$

and the proposition follows from the definition of the circular units $\alpha(T)$ and $\alpha(T l)$. 
Proposition $5.2 \alpha(T l) \equiv \sigma_{l, T}^{-1} \alpha(T) \quad(\bmod \lambda)$, where $\lambda$ is any prime of $K_{T l}$ above $l$.

Proof: This follows from equation (29) together with the fact that $a$ is an inverse for $l$ in $(\mathbf{Z} / T \mathbf{Z})^{*}$ and that $\zeta_{l} \equiv 1 \quad(\bmod \lambda)$.

Propositions 5.1 and 5.2 make up the axioms of an Euler system in the sense of Kolyvagin [Ko].

\section{Divisibility properties of the circular units}

In addition to the norm operator $\mathbf{N}_{l}$ defined in the previous section, the following derivative operators in the group ring $\mathbf{Z}\left[\Gamma_{S}\right]$ are a key ingredient in Kolyvagin and Thaine's method. For each prime $l$ in $\mathcal{S}$, choose a generator $\gamma_{l}$ for $\Gamma_{l}$ and let

$$
\mathrm{D}_{l}=\sum_{i=1}^{l-2} i \gamma_{l}^{i}, \quad \mathrm{D}_{T}=\prod_{l \mid T} \mathrm{D}_{l},
$$

the product being taken in the group ring $\mathbf{Z}\left[\Gamma_{T}\right]$.

Lemma $6.1\left(\gamma_{l}-1\right) D_{l}=(l-1)-\mathbf{N}_{l}$.

Proof: A direct computation.

The group ring $\mathbf{Z}\left[\Gamma_{T}\right]$ operates on the group $K_{T}^{*}$ in a natural way. Let

$$
\beta(T)=\mathrm{D}_{T} \alpha(T) \in K_{T}^{*},
$$

and let $n(T)$ be the largest odd divisor of $\operatorname{gcd}_{l \mid T}(l-1)$.

From now on, we will assume that $T$ is a product of primes which are split in $K / \mathbf{Q}$. Although $\beta(T)$, unlike $\mathbf{N}_{T} \alpha(T)$, need not be invariant under the action of $\Gamma_{T}$, it is invariant modulo $n(T)$-th powers.

Lemma $6.2 \beta(T)$ belongs to $\left(K_{T}^{*} / K_{T}^{* n(T)}\right)^{\Gamma_{T}}$.

Proof: By induction on the number of primes dividing T. Assume the lemma for all proper divisors of $T$, and write $T=l Q$. Modulo $n(T)$, one has:

$$
\begin{aligned}
\left(\gamma_{l}-1\right) \mathrm{D}_{T} \alpha(T) & =\left(l-1-\mathrm{N}_{l}\right) \mathrm{D}_{Q} \alpha(T) \quad \text { (lemma 6.1) } \\
& =\left(\sigma_{l, Q}^{-1}-1\right) \mathrm{D}_{Q} \alpha(Q) \quad \text { (prop. 5.1) } \\
& =0 \text { by the induction hypothesis. }
\end{aligned}
$$


In the last step we use the fact that $\sigma_{l, Q}=1$ in $\operatorname{Gal}(K / Q)$, so that $\sigma_{l, Q}$ belongs to $\Gamma_{Q}$.

Lemma 6.3 The natural map $K^{*} / K^{* n(T)} \longrightarrow\left(K_{T}^{*} / K_{T}^{* n(T)}\right)^{\Gamma_{T}}$ is an isomorphism.

Proof: The group of $n(T)$-th roots of unity in $K_{T}$ is trivial. Hence the sequence

$$
1 \longrightarrow K_{T}^{*} \stackrel{n(T)}{\longrightarrow} K_{T}^{*} \longrightarrow K_{T}^{*} / K_{T}^{* n_{T}} \longrightarrow 1
$$

is exact. Taking $\Gamma_{T}$-invariants gives rise to the cohomology exact sequence

$$
1 \longrightarrow K^{*} / K^{* n(T)} \longrightarrow\left(K_{T}^{*} / K_{T}^{* n(T)}\right)^{\Gamma_{T}} \longrightarrow H^{1}\left(\Gamma_{T}, K_{T}^{*}\right)_{n(T)} \longrightarrow 1,
$$

and the lemma follows from Hilbert's theorem $90\left(H^{1}\left(\Gamma_{T}, K_{T}^{*}\right)=0\right)$.

Let $\kappa(T)$ denote the preimage of $\beta(T)$ by this isomorphism. For each prime $l$ in $\mathcal{S}$, choose a place $\lambda$ of $K$ above it. Write

$$
v_{\lambda}: K^{*} \longrightarrow \mathbf{Z}
$$

for the valuation map at $\lambda$, and $\tilde{v}_{\lambda}$ for the induced map on $K^{*} / K^{* n(T)}$, making the following diagram commute:

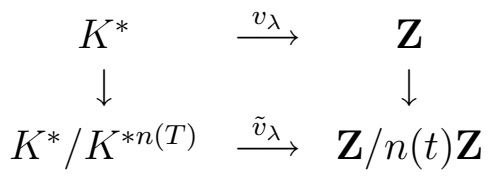

Let $u_{l}$ denote the image of $\gamma_{l}$ by the isomorphism $\Gamma_{l} \longrightarrow(\mathbf{Z} / l \mathbf{Z})^{*}$. Given $\kappa$ in $K^{*}$, let $\operatorname{red}_{\lambda}(\kappa) \in k_{\lambda}^{*}$ be the reduction of $\kappa \bmod \lambda$, in the residue field $k_{\lambda}=\mathbf{Z} /$ lZZ. Finally, let

$$
\log _{u_{l}}: k_{\lambda}^{*} \longrightarrow \mathbf{Z} /(l-1) \mathbf{Z}
$$

be the logarithm map to the base $u_{l}$. The following proposition contains the information that we will need on the ideal factorization of the $\kappa(T)$.

\section{Proposition 6.4}

1. If $l$ does not divide $T$, then $\tilde{v}_{\lambda}(\kappa(T))=0$. 
2. If $l$ is split in $K_{T} / \mathbf{Q}$, then

$$
\tilde{v}_{\lambda}(\kappa(T l))=-\log _{u_{l}}\left(\operatorname{red}_{\lambda}(\kappa(T))\right) \quad(\bmod n(T l)) .
$$

Proof:

1. If $l$ does not divide $T$, then $\lambda$ is unramified in $K_{T} / K$, and hence the valuation map $\tilde{v}_{\lambda}$ extends from $K^{*} / K^{* n(T)}$ to $K_{T}^{*} / K_{T}^{* n(T)}$. But clearly $\tilde{v}_{\lambda}(\beta(T))=$ 0 , since $\beta(T)$ is a unit in $K_{T}^{*}$.

2. Let $\lambda^{\prime}$ be a prime of $K_{T}$ above $\lambda$, and let $\lambda^{\prime \prime}$ be the prime of $K_{T l}$ above $\lambda^{\prime}$. Let $v_{\lambda^{\prime}}\left(\right.$ resp. $\left.v_{\lambda^{\prime \prime}}\right)$ be the valuations on $K_{T}\left(\operatorname{resp} K_{T l}\right)$ normalized to be 1 on uniformizing elements, so that

$$
v_{\lambda^{\prime}}(\kappa)=\frac{1}{l-1} v_{\lambda^{\prime \prime}}(\kappa), \quad \kappa \in K_{T}^{*}
$$

Writing

$$
\kappa(T l)=\beta(T l) \rho^{-n(T l)}, \quad \rho \in K_{T l},
$$

and using the fact that $\beta(T l)$ is a unit, one finds

$$
v_{\lambda}(\kappa(T l))=-\frac{n(T l)}{l-1} v_{\lambda^{\prime \prime}}(\rho) .
$$

By definition of $u_{l}$, one has

$$
v_{\lambda^{\prime \prime}}(\rho)=\log _{u_{l}}\left(\operatorname{red}_{\lambda^{\prime \prime}}\left(\left(\gamma_{l}-1\right) \rho\right)\right) \quad(\bmod l-1) .
$$

But

$$
\begin{aligned}
\left(\gamma_{l}-1\right) \rho & =\frac{1}{n(T l)}\left[\left(\gamma_{l}-1\right) \beta(T l)\right] \\
& =\frac{1}{n(T l)}\left[(l-1) \mathrm{D}_{T} \alpha(T l)+\left(1-\sigma_{l, T}^{-1}\right) \mathrm{D}_{T} \alpha(T)\right] \\
& =\frac{l-1}{n(T l)} \mathrm{D}_{T} \alpha(T l), \quad \text { since } \sigma_{l, T}=1
\end{aligned}
$$

Hence by prop. 5.2,

$$
\operatorname{red}_{\lambda^{\prime \prime}}\left(\left(\gamma_{l}-1\right) \rho\right)=\operatorname{red}_{\lambda^{\prime}}\left(\frac{l-1}{n(T l)} \mathrm{D}_{T} \alpha(T)\right),
$$


and hence

$$
\log _{u_{l}} \operatorname{red}_{\lambda^{\prime \prime}}\left(\left(\gamma_{l}-1\right) \rho\right) \equiv \log _{u_{l}} \operatorname{red}_{\lambda^{\prime}}\left(\frac{l-1)}{n(T l)} \beta(T)\right) \quad(\bmod l-1) .
$$

Combining equations (38), (39) and (41), one obtains

$$
\tilde{v}_{\lambda}(\kappa(T l)) \equiv-\log _{u_{l}}\left(\operatorname{red}_{\lambda} \kappa(T)\right) \quad(\bmod n(T l))
$$

as desired.

If $M$ is a $\mathbf{Z}$-module and $m$ belongs to $M$, we say that $n \in \mathbf{Z}$ divides $m$ if there exists $m^{\prime} \in M$ with $n \cdot m^{\prime}=m$. Given a rational prime $p$, one defines $\operatorname{ord}_{p}(m)$ to be the integer $M$ such that $p^{M}$ divides $m$, but $p^{M+1}$ does not. (If this integer does not exist one sets $\operatorname{ord}_{p}(m)=\infty$.) Recall that $C_{S}(K)$ is defined to be the quotient of the ideal class group of $K$ by the subgroup generated by the prime ideals lying above $S$, and that $h_{S}(K)$ denotes its order. The main result of Thaine and Kolyvagin gives a bound on the order of $C_{S}(K)$ in terms of the divisibilty of the elements $\kappa(S)$.

Theorem 6.5 (Thaine, Kolyvagin) The greatest common divisor of $n(S)$ and $h_{S}(K)$ divides $\kappa(S)$.

Proof: We prove this by induction on $h_{S}(K)$. If $h_{S}(K)=1$, then the theorem is trivially true. Otherwise, choose a prime $p$ dividing $h_{S}(K)$. Suppose that $\operatorname{ord}_{p}(\kappa(S))=M_{0}<\infty$, and let $M=M_{0}+1$. We must whow that $p^{M}$ does not divide $\operatorname{gcd}\left(n(S), h_{S}(K)\right)$. If $p^{M}$ does not divide $n(S)$, we are done. Hence, suppose that $p^{M}$ divides $n(S)$. (So that in particular, $p$ is odd). Now, choose a prime $l$ in $\mathcal{S}$ not dividing $S$, such that

1. $l$ splits in $K / \mathbf{Q}$; let $\lambda$ denote a prime of $K$ lying above it.

2. $l \equiv 1 \quad\left(\bmod P^{M}\right)$ (i.e., $l$ splits in $\left.\mathbf{Q}\left(\mu_{p^{M}}\right) / \mathbf{Q}\right)$.

3. $\operatorname{ord}_{p}\left(\operatorname{red}_{\lambda}(\kappa(S))\right)=M_{0}$.

4. The image of $\lambda$ in $C_{S}(K) \otimes \mathbf{Z}_{p}$ is non trivial, and the exact sequence

$$
0 \longrightarrow\langle\lambda\rangle \longrightarrow C_{S}(K) \otimes \mathbf{Z}_{p} \longrightarrow C_{S l}(K) \otimes \mathbf{Z}_{p} \longrightarrow 0
$$

is split (and hence in particular $\operatorname{ord}_{p}(\lambda)=0$ ). 
Let $F=K\left(\mu_{p^{M}}, \kappa(S)^{1 / p^{M}}\right)$. Conditions 2 and 3 are equivalent to the condition that $\operatorname{Frob}_{\lambda}$ in $\operatorname{Gal}(F / K)$ belongs to the subgroup $\operatorname{Gal}\left(F / K\left(\mu_{p^{M}}\right)\right)$ and is non-trivial. Condition 4 is equivalent to a condition on $\operatorname{Frob}_{\lambda}$ in $\operatorname{Gal}\left(H_{S} / K\right)$ where $H_{S}$ is a non-trivial subfield of the Hilbert class field $H$ of $K$. Since $F$ and $H$ are linearly disjoint over $K$ (as can be seen for example by ramification considerations), it follows from the Chebotarev density theorem that conditions 1-4 can be imposed simultaneously.

Let $m=\operatorname{ord}_{p}(\kappa(S l))$. By combining proposition 6.4 with condition 3 satisfied by $l$, one has

$$
\operatorname{ord}_{p}\left(\tilde{v}_{\lambda}(\kappa(S l))\right)=M_{0},
$$

and hence a fortiori $m \leq M_{0}$. Moreover, since $p^{M}$ divides $l-1$, it also divides $n(S l)$. Let $\rho$ be the natural projection of $\kappa(S l)$ to $K^{*} / K^{* p^{M}}$, and let $\kappa^{\prime}(S l)=\rho^{1 / p^{m}}$ which is well defined in $K^{*} / K^{* p^{M-m}}$. By equation 43 and condition 3 , one has

$$
\tilde{v}_{\lambda}\left(\kappa^{\prime}(S l)\right)=u \cdot p^{M_{0}-m},
$$

where $u$ is a unit in $\mathbf{Z} / p^{M-m} \mathbf{Z}$. Hence $p^{M_{0}-m}$ annihilates the class of $\lambda$ in $C(S) \otimes \mathbf{Z} / p^{M-m} \mathbf{Z}$. Because of condition 4 , we have

$$
\#\langle\lambda\rangle \leq p^{M_{0}-m} .
$$

In particular, $m<M_{0}$, and by the induction hypothesis,

$$
\# C_{S l}(K) \otimes \mathbf{Z}_{p} \leq p^{m} .
$$

Combining the inequalities (45) and (46) gives

$$
\# C_{S}(K) \otimes \mathbf{Z}_{p} \leq p^{M_{0}},
$$

so that $p^{M}$ does not divide $h_{S}(K)$, as was to be shown.

\section{$7 \quad$ Formal properties of $\theta^{\prime}(\omega, S)$}

We now turn to the study of the element $\theta^{\prime}(\omega, S)$ defined by

$$
\theta^{\prime}(\omega, S)=\sum_{\sigma \in \Gamma_{S}} \sigma \alpha(S) \otimes \sigma \in K_{S}^{*} \otimes \mathbf{Z}\left[\Gamma_{S}\right] .
$$


Given

$$
\gamma \in \operatorname{Gal}\left(K_{S} / \mathbf{Q}\right)=\Gamma_{S} \times \operatorname{Gal}(K / \mathbf{Q}),
$$

let $\gamma(T)$ denote its natural projection in $\Gamma_{T}$.

The group $\operatorname{Gal}\left(K_{S} / \mathbf{Q}\right)$ acts on the left of $K_{S}^{*} \otimes \mathbf{Z}\left[\Gamma_{S}\right]$ by the Galois action, and $\Gamma_{S}$ acts on the right by multiplication in the group ring.

Lemma $7.1 \gamma \theta^{\prime}(\omega, S)=\omega(\gamma) \cdot \theta^{\prime}(\omega, S) \cdot \gamma(S)^{-1}$.

Proof: A change of variable argument.

Given a divisor $T$ of $S$, let $P_{S, T}: K_{S}^{*} \otimes \mathbf{Z}\left[\Gamma_{S}\right] \longrightarrow K_{S}^{*} \otimes \mathbf{Z}\left[\Gamma_{S}\right]$ be the map induced by the projection $\Gamma_{S} \longrightarrow \Gamma_{T} \subset \Gamma_{S}$.

\section{Lemma 7.2}

$$
P_{S, T}\left(\theta^{\prime}(\omega, S)\right)=\theta^{\prime}(\omega, T) \cdot \prod_{l \mid S / T}\left(1-\omega(l) \cdot \sigma_{l, T}\right)
$$

Proof: One has

$$
P_{S, T}\left(\theta^{\prime}(\omega, S)\right)=\sum_{\sigma \in \Gamma_{T}}\left(\mathbf{N}_{S / T} \cdot \sigma \alpha_{S} \otimes \sigma\right)
$$

Hence by proposition 5.1

$$
P_{S, T}\left(\theta^{\prime}(\omega, S)\right)=\left(\prod_{l \mid S / T}\left(1-\sigma_{l, T}^{-1}\right)\right) \theta^{\prime}(\omega, T),
$$

which is equal to $\theta^{\prime}(\omega, T) \cdot \Pi\left(1-\omega(l) \sigma_{l, T}\right)$ by lemma 7.1 .

\section{The order of vanishing of $\theta^{\prime}(\omega, S)$}

Let us write $S$ as $S=P Q$, where $P=l_{1} \cdots l_{s}$ is a product of split primes in $K / \mathbf{Q}$, and $Q$ is a product of inert primes. When $\sigma$ runs over $\Gamma_{S}$, write

$$
\sigma=\sigma_{1} \cdots \sigma_{s} \tau,
$$

for its unique decomposition as a product with $\sigma_{i} \in \Gamma_{l_{i}}$, and $\tau \in \Gamma_{Q}$. 


\section{Lemma 8.1}

$$
\begin{aligned}
\theta^{\prime}(\omega, S)= & \sum_{\sigma \in \Gamma_{S}} \sigma \alpha_{S}\left(\sigma_{1}-1\right) \cdots\left(\sigma_{s}-1\right) \tau \\
& \quad-\sum_{T \mid P, T \neq P}\left(\mu(P / T) \cdot \theta^{\prime}(\omega, T Q) \cdot \prod_{l \mid P / T}\left(1-\sigma_{l, T Q}\right)\right) .
\end{aligned}
$$

Proof: By direct computation,

$$
\sum_{\sigma \in \Gamma_{S}} \sigma \alpha_{S}\left(\sigma_{1}-1\right) \cdots\left(\sigma_{s}-1\right) \tau=\theta^{\prime}(\omega, S)+\sum_{T \mid, T \neq P} \mu(P / T) P_{S, T Q}\left(\theta^{\prime}(\omega, S)\right) .
$$

The formula now follows from lemma 7.2.

We are now ready to prove theorem 4.2.

Theorem 4.2 (Order of vanishing) The element $\theta^{\prime}(\omega, S)$ belongs to $K_{S}^{*} \otimes$ $I^{s}=K_{S}^{*} \otimes I^{r-1}$.

Proof: By induction on $s$, using lemma 8.1 for the induction step.

\section{The leading coefficient}

We now turn to the study of the element $\tilde{\theta}^{\prime}(\omega, S)$ defined by projecting $\theta^{\prime}(\omega, S)$ to the value group $K_{S}^{*} \otimes\left(I_{2}^{r-1} / I_{2}^{r}\right)$.

Lemma 9.1 The leading coefficient $\tilde{\theta}^{\prime}(\omega, S)_{2}$ belongs to the subgroup of elements in $\left(K_{S}^{*} \otimes\left(I_{2}^{r-1} / I_{2}^{r}\right)\right)^{\Gamma_{S}}$ fixed by the left (Galois) action of $\Gamma_{S}$.

Proof: Given $\sigma$ in $\Gamma_{S}$, by lemma 7.1 we have

$$
(\sigma-1) \tilde{\theta}^{\prime}(\omega, S)_{2}=\tilde{\theta}^{\prime}(\omega, S)_{2}\left(\sigma^{-1}-1\right),
$$

and lemma 9.1 follows.

Lemma 9.2 Let $\Gamma$ be a finite abelian group of odd order, and let $\Gamma_{S}$ act on the module $K_{S}^{*} \otimes \Gamma$ by the Galois action. Then the natural map

$$
K^{*} \otimes \Gamma \longrightarrow\left(K_{S}^{*} \otimes \Gamma\right)^{\Gamma_{S}}
$$

is an isomorphism. 
Proof: By decomposing $\Gamma$ as a direct product of cyclic groups, one reduces the proof of lemma 9.2 to the case where $\Gamma$ is cyclic of odd order $n$. If $K_{S}$ contains no $n$-th roots of unity, then we are in the situation of lemma 6.3. In general, one uses the fact that the restriction map

$$
H^{1}\left(K, \mu_{n}\right) \longrightarrow H^{1}\left(K_{S}, \mu_{n}\right)^{\Gamma_{S}}
$$

is an isomorphism.

Lemma $9.3 n(S)\left(\gamma_{l_{1}}-1\right) \cdots\left(\gamma_{l_{s}}-1\right)=0 \quad\left(\bmod I_{2}^{r}\right)$.

Proof: We can write $\left(\gamma_{l_{1}}-1\right) \cdots\left(\gamma_{l_{s}}-1\right)$ as a sum of terms of the form $\left(\gamma_{l_{1}}^{(p)}-1\right) \cdots\left(\gamma_{l_{s}}^{(p)}-1\right) \quad\left(\bmod I_{2}^{r}\right)$, where the $\gamma_{l_{i}}^{(p)}$ are of order a power of $p(p$ an odd prime) and at least one of the $\gamma_{l_{j}}^{(p)}$ is of order exactly $q=p^{\operatorname{ord}_{p}(n(S))}$. Hence it suffices to show the theorem when $n(S)=q$ is a power of a prime. In that case, one has

$$
0=\gamma_{l_{j}}^{q}-1=\sum_{i=1}^{q}\left(\begin{array}{c}
q \\
i
\end{array}\right)\left(\gamma_{l_{j}}-1\right)^{i}
$$

so that $q\left(\gamma_{l_{j}}-1\right) \in I_{2}^{2}$. The result follows.

The following proposition gives an inductive formula for the leading coefficient $\tilde{\theta}^{\prime}(\omega, S)_{2}$.

\section{Proposition 9.4 .}

$$
\begin{aligned}
\tilde{\theta}^{\prime}(\omega, S)_{2}= & 2^{\#(l \mid Q)} \kappa(P) \otimes\left(\gamma_{l_{1}}-1\right) \cdots\left(\gamma_{l_{s}}-1\right) \\
& -\sum_{T \mid P, T \neq P} \mu(P / T) \cdot \tilde{\theta}^{\prime}(\omega, T) \cdot \prod\left(1-\sigma_{l, T}\right) .
\end{aligned}
$$

Proof: This follows from lemma 8.1 together with the fact that

$$
\sum_{\sigma \in \Gamma_{S}} \sigma \alpha_{S} \otimes\left(\sigma_{1}-1\right) \cdots\left(\sigma_{s}-1\right) \tau=2^{\#(l \mid Q)} \beta(P) \otimes\left(\gamma_{l_{1}}-1\right) \cdots\left(\gamma_{l_{s}}-1\right)
$$

in $K_{S}^{*} \otimes\left(I^{r-1} / I^{r}\right)$. Because $\left(\gamma_{l_{1}}-1\right) \cdots\left(\gamma_{l_{s}}-1\right)$ is killed by $n(P)$ in $I_{2}^{r-1} / I_{2}^{r}$ (lemma 9.3), one can replace $\beta(P)$ by $\kappa(P)$ in the formula.

In the remainder of this section we will prove theorem 4.5 which we first recall: 


\section{Theorem 4.5}

1. Conjecture 4.3 is true when $r=1$.

2. $\tilde{\theta}^{\prime}(\omega, S)_{2}$ belongs to $K^{*} \otimes I_{2}^{r-1} / I_{2}^{r}$.

3. If $\operatorname{gcd}\left(h_{S}(K), n(T)\right)=1$ for all $T \mid S$, then $\tilde{\theta}^{\prime}(\omega, S)_{2}$ belongs to $\mathcal{O}_{s}^{*} \otimes$ $\left(I_{2}^{r-1} / I_{2}^{r}\right)$.

4. $h_{S}(K)$ divides $\tilde{\theta}^{\prime}(\omega, S)_{2}$.

5. Suppose that $\Gamma_{S}=\Gamma_{l}$ is cyclic, and that $l$ is split in $K / \mathbf{Q}$ so that $r=2$. Let $\lambda$ be a prime of $K$ above $l$, and let $k_{\lambda} \simeq \mathbf{F}_{l}$ denote the residue field at $\lambda$. If the fundamental unit of $K / \mathbf{Q}$ is a generator for $k_{\lambda}^{*}$, and $\operatorname{gcd}(h(K), n(l))=1$, then

$$
\tilde{\theta}^{\prime}(\omega, l)_{2}= \pm 2 h_{l} R_{l} \quad\left(\bmod I_{2}^{2}\right) .
$$

Proof:

1. When $r=1$, we have $\tilde{\theta}^{\prime}(\omega, S)=P_{S, 1}\left(\theta^{\prime}(\omega, S)\right)$, where $P_{S, 1}: \mathbf{Z}\left[\Gamma_{S}\right] \longrightarrow$ $\mathbf{Z}$ is the augmentation map. By lemma 7.2,

$$
P_{S, 1}\left(\theta^{\prime}(\omega, S)\right)=\alpha(1) \prod_{l \mid S}(1-\omega(l))=2^{\#(l \mid S)} \alpha(1),
$$

since all the $l$ dividing $S$ are inert in $K / \mathbf{Q}$. We know from Dirichlet's analytic class number formula that $\alpha(1)=2 h_{1} R_{1}$, and hence the result follows.

2. Combine lemmas 9.1 and 9.2.

3. By prop 6.4, we have $v_{\lambda}(\kappa(T))=0 \quad(\bmod n(T))$ for all places $\lambda$ which do not lie above $S$. Let $\left(K^{*} / K^{* n(T)}\right)(S)$ denote the subgroup of elements in $K^{*} / K^{* n(T)}$ satisfying this property. There is a natural exact sequence

$$
0 \longrightarrow \mathcal{O}_{S}^{*} / \mathcal{O}_{S}^{* n(T)} \longrightarrow\left(K^{*} / K^{* n(T)}\right)(S) \longrightarrow C_{S}(K) \otimes \mathbf{Z} / n(T) \mathbf{Z} .
$$

The assumption that $\left(h_{S}(K), n(T)\right)=1$ for all $T \mid S$ implies that the natural map from $\mathcal{O}_{S}^{*} / \mathcal{O}_{S}^{* n(T)}$ to $\left(K^{*} / K^{* n(T)}\right)(S)$ is an isomorphism, so that the $\kappa(T)$ are $S$-units modulo $n(T)$-th powers. The result follows from prop. 9.4. 
4. This is a direct consequence of theorem 6.5 combined with prop. 9.4.

5. The fact that $\operatorname{gcd}\left(h_{l}(K), n(l)\right)=1$ implies, by the previous fact, that $\kappa(l)$ is an $l$-unit of $K$ modulo $n(l)$ th powers, and hence $\tilde{\theta}^{\prime}(\omega, l)$ belongs to $\mathcal{O}_{l}^{*} \otimes\left(I_{2} / I_{2}^{2}\right)$. We want to prove the equality of two objects in $\mathcal{O}_{l}^{*} \otimes\left(I / I^{2}\right)$. For this, we use two maps:

$$
\phi_{1}: \mathcal{O}_{l}^{*} \otimes\left(I_{2} / I_{2}^{2}\right) \longrightarrow I_{2} / I_{2}^{2}, \quad \phi_{2}: \mathcal{O}_{l}^{*} \otimes\left(I_{2} / I_{2}^{2}\right) \longrightarrow I_{2}^{2} / I_{2}^{3} .
$$

The first is induced from the map $v_{\lambda}: \mathcal{O}_{l}^{*} \longrightarrow \mathbf{Z}$, and the second from the map $\operatorname{rec}_{\lambda}: \mathcal{O}_{l}^{*} \longrightarrow \Gamma_{l} \longrightarrow I_{2} / I_{2}^{2}$ given by the reciprocity law of local class field theory. Because $\operatorname{gcd}(h(K), n(l))=1$, the kernel of the map $\phi_{1}$ is just $\mathcal{O}_{K}^{*} \otimes\left(I_{2} / I_{2}^{2}\right)$. The assumption that the fundamental unit for $K$ is a generator of $k_{\lambda}^{*}$ means that $\phi_{2}$ is injective on $\mathcal{O}_{K}^{*} \otimes\left(I_{2} / I_{2}^{2}\right)$. Hence, if two elements in $\mathcal{O}_{l}^{*} \otimes\left(I_{2} / I_{2}^{2}\right)$ have the same image by $\phi_{1}$ and $\phi_{2}$, then they are equal.

Recall that $u_{l} \in k_{\lambda}^{*}$ denotes the element which corresponds to the chosen generator $\gamma_{l}$ of $\Gamma_{l}$ by the reciprocity law of local class field theory. By prop. 9.4, we have

$$
\tilde{\theta}^{\prime}(\omega, l)_{2}=\kappa(l) \otimes\left(\gamma_{l}-1\right) .
$$

Hence, by prop. 6.4,

$$
\phi_{1}\left(\tilde{\theta}^{\prime}(\omega, l)_{2}\right)=v_{\lambda}(\kappa(l)) \otimes\left(\gamma_{l}-1\right)=\log _{u_{l}}(\kappa(1))\left(\gamma_{l}-1\right) .
$$

Let $u$ be a fundamental unit for $K$. By Dirichlet's class number formula, we can write

$$
\kappa(1)=u^{ \pm 2 h}
$$

so that $\log _{u_{l}}(\kappa(1))= \pm 2 h \log _{u_{l}}(u)$. It follows that

$$
\phi_{1}\left(\tilde{\theta}^{\prime}(\omega, l)_{2}\right)= \pm 2 h \log _{u_{l}}(u)\left(\gamma_{l}-1\right)= \pm 2 h(\operatorname{rec}(u)-1) .
$$

Since $\kappa(l)=\beta(l) x^{n(l)}$, where $x$ belongs to $K_{l}^{*}$, and since

$$
\operatorname{norm}_{K_{l} / K}(\beta(l))=1
$$

by prop. 5.1 , we have by taking norms:

$$
\kappa(l)^{l-1}=\operatorname{norm}_{K_{l} / K} x^{n(l)} .
$$


Hence $\kappa(l)^{(l-1) / n(l)}= \pm$ norm $_{K_{l} / K} x$, so that $\kappa(l)^{2^{a}}$ is a norm for some $a \geq 0$. Since norms lie in the kernel of the local reciprocity map, we find that

$$
\phi_{2}\left(\tilde{\theta}^{\prime}(\omega, l)_{2}\right)=0 .
$$

We choose a $\mathbf{Z}$-basis for $\mathcal{O}_{l}^{*}$, given by a fundamental unit $u$ and an $l$-unit $u(l)$. This can be done in such a way that

$$
v_{\lambda}(u(l))=h / h_{l},
$$

since this number is the order of the class of $\lambda$ in the ideal class group of $K$. The regulator $R_{l}$ can be written explicitly as

$$
R_{l}= \pm(u \otimes(\operatorname{rec}(u(l))-1)-u(l) \otimes(\operatorname{rec}(u)-1)) .
$$

Hence,

$$
\phi_{1}\left(2 h_{l} R_{l}\right)= \pm 2 h_{l} v_{\lambda}(u(l)) \otimes(\operatorname{rec}(u)-1)= \pm 2 h(\operatorname{rec}(u)-1) .
$$

It is immediate from the definition of $R_{l}$ that

$$
\phi_{2}\left(4 h_{l} R_{l}\right)=0 \text {. }
$$

Combining equations (62), (64), (67), and (68) we find that

$$
\tilde{\theta}^{\prime}(\omega, l)_{2}= \pm 2 h_{l} R_{l} \quad\left(\bmod I_{2}^{2}\right),
$$

as claimed.

\section{References}

[DR] P. Deligne and K. Ribet, Values of abelian L-functions at negative integers over totally real fields, Invent. Math. 59 (1980), pp. 227286.

[Gr] B.H. Gross, On the values of abelian L-functions at $s=0$, Journal of the Faculty of Science, the University of Tokyo, Sect. IA, Math. Vol. 35, No. 1, pp. 177-197, March, 1988. 
[H1] Alfred W. Hales, Augmentation terminals of finite abelian groups, in SLN 1006, 1983, pp. 720-733.

[H2] Alfred W. Hales, Stable augmentation quotients of abelian groups, Pacific Journal of Math, vol 118, no. 2, 1985, pp. 401-410.

[Iw] K. Iwasawa, On $\mathbf{Z}_{l}$-extensions of algebraic number fields, Annals of Mathematics, second series, vol. 98, no. 2, 1973.

[Ko] V.A. Kolyvagin, Euler Systems, (1988). To appear in a Birkhäuser volume in honor of Grothendieck.

[La] S. Lang, Cyclotomic fields I and II, combined second edition, GTM 121, Springer-Verlag 1990.

[MT] B. Mazur and J. Tate, Refined conjectures of the "Birch and Swinnerton-Dyer type", Duke Math Journal, Vol. 54, No. 2, 1987, p. 711.

[Pa] I.B.S. Passi, Group rings and their augmentation ideals, SLN 715, Berlin-New York, 1979.

[Ru] K.C. Rubin, Global units and ideal class groups, Invent. Math. 89, 511-526, (1987).

[Sch] R. Schoof, preprint.

[Th] F. Thaine, On the ideal class groups of real abelian number fields, Ann. of Math. 128 (1988), pp. 1-18.

[Wa] L. Washington, Introduction to cyclotomic fields, GTM 83, New York, Springer-Verlag (1982). 